\title{
Akademische Existenzgründungen in der Internetbranche - welchen Einfluss haben Hochschule und Region auf die Standortwahl?
}

\author{
Johannes Kopper ${ }^{1}$ Angelika Jäger ${ }^{2}$
}

Eingegangen: 12. Oktober 2015 / Angenommen: 28. September 2016/ Online publiziert: 18. Oktober 2016

() Springer-Verlag Berlin Heidelberg 2016

Zusammenfassung Eine regionale Ausrichtung der Forschungsaktivität und die wirtschaftliche Unterstützung der Standortregion werden zunehmend als erweiterte Aufgabe des Hochschulwesens angesehen. Der Beitrag untersucht, ob und in welchem Ausmaß Akademiker aus der Internetbranche innerhalb der Region ihrer Alma Mater gründen und welche hochschul- und regionsseitigen Faktoren diese Entscheidung beeinflussen. Hängt es verstärkt von der Region ab, ob Gründer verbleiben oder in anderen Regionen gründen? Welchen Einfluss haben Struktur und Strategie der Hochschule? Dies ist für politische Entscheidungsträger und Hochschulakteure gleichermaßen von Interesse, da die Regionalwirksamkeit von Spin-offs und damit ihr Beitrag zum regionalwirtschaftlichen Geschehen in Frage gestellt wird. Diese Regionalwirksamkeit ist es, die eine hohe finanzielle Gründerförderung aus öffentlicher Hand begründet. Die Studie basiert auf der digitalen Plattform „Gründerszene“, die Dienstleistungen für Gründer im Internetbereich anbietet. Mittels eines Regressionsansatzes wer-

Zusatzmaterial online Zusätzliche Informationen sind in der Online-Version dieses Artikels (doi: 10.1007/s13147-016-0439-y) enthalten.

Das Zusatzmaterial enthält eine Korrelationsmatrix der erklärenden Variablen, die in unsere Modelle integriert wurden

Johannes Kopper, M.A.

johannes.kopper@hs-niederrhein.de

Angelika Jäger, M.A.

angelika.jaeger@nielsen.com

1 Niederrhein Institut für Regionalund Strukturforschung (NIERS),

Richard-Wagner-Straße 140, 41065 Mönchengladbach, Deutschland

2 The Nielsen Company GmbH, Sachsenstraße 16, 20097 Hamburg. Deutschland den hochschul- und regionsseitige Einflussfaktoren auf die Standortwahl der betrachteten Gründer identifiziert. Dabei zeigt sich als prägnantes Ergebnis, dass in wissens-, innovations- und wirtschaftlich leistungsstarken Regionen keine höhere Verbleibsquote festzustellen ist als in strukturschwachen Regionen, vielmehr kommt es auf die Existenz eines regionalen Gründungsklimas an.

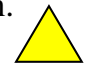

Schlüsselwörter Start-Ups · Regionaler Wissenstransfer · Internetbranche

\section{Internet Start-Ups - which Influence do the Alma Mater and the University Region have on the Location Decision of Founders?}

Abstract The regional orientation of academic activities and economic support of the university region are increasingly important responsibilities. The paper examines to which extent academic founders of the online sector decide to establish firms close to their alma mater, and which factors affecting this decision are associated with the university or the region. Are entrepreneurs' decisions to stay or leave the region dependent upon factors of the region itself? What influence do the structure and strategic focus of the university have on this decision? These questions are of interest for political decision makers and university stakeholders - especially as the regional effectiveness of start-ups and their contribution to regional economy is put into question. This regional effectiveness justifies high public financial support. The analysis is based on the German digital platform Gxünderszene, which provides services for founders in the be sector. By using regression analysis, the factors of influence of academic institutions and the region on the location decision of online founders are iden- 
tified. There is no noticeably higher rate of founders deciding to stay within regions which are strong with knowledge, innovation, and service compared to structurally weak regions. On the other hand, a regional entrepreneurial climate that is conducive for start-ups is very important.

Keywords Start-up. Regional knowledge transfer. Online sactor

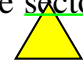

\section{Einleitung}

Die Third Mission ${ }^{1}$ des Hochschulwesens rückt ergänzend zu den tr ionellen Funktionen Lehre und Forschung verstärkt in den Fokus öffentlicher Aufmerksamkeit und politikberatender Forschung (Caniëls/van den Bosch 2Q11; Roessler/Duong/Hachmeister 2Q15). Hochschulen wi in Potenzial des sozialwirtscha hen Engagements zugesprochen, in dessen Rahmen sie als Erbauer und wichtige Akteure eines regionalen Innovationsystems (Regional System Builder) (Caniëls/van den Bosch 2011: 272) und als Unterstützer des regionalen sozialök bischen Systems agieren. Ein Instrument zur Erhöhung des regionalen Wissenstransfers ist die Unterstützung von Hochschulausgründungen. In der Wissenstransferforschung (und in diesem Beitrag) wendet sich die Aufmerksamkeit daher akademischen Spin-offs zu. Das sind Gründungen, bei denen Wissen angewendet wird, das in der Hochschule im Rahmen von $\$$ chung oder akademischer Bildung erworben wurde. Spin-offs gelten als Leistungsindikatoren für die Innovationsrelevanz der Hochschule (Schmoch/Licht/Reinhard 2000: 52). Sie agieren als Brücke zwischen Hochschulforschung und Wirtschaft, indem sie die Weiterentwicklung wissenschaftlicher Ausbildungs- und Forschungsergebnisse zu einem marktfähigen Produkt und somit ihre wirtschaftliche Nutzbarkeit fördern (Wissenschaftsrat 2007; Hamm/ Jäger/Karl et al. 2Q13a: 71).

Für die Regi Jökonomie sind Spin-offs von hohem Interesse, da sie sich oft nahe der Alma Mater ansiedeln (Schmude 1894: 77; Egeln/Gottschalk/Rammer et al. 2h02: 38; Schleid Ser 2006: 63; Fritsch/Henning/Slavtchey 1 l. 2007: 164), um Kontakten und Infrastruktur der Hochschule sowie vom Hochschulnetzwerk zu profitieren. Somit besitzen Spin-offs das Potenzial, auf das regionale Innovationsgeschehen einzuwirken, Wertschöpfungseffekte auszulösen, den Strukturwandel zu unterstützen und die ökonomische Entwicklung der Region zu fördern. Akademische

1 Dieser .,dritte Auftrag“ beinhaltet unter anderem. Hochschulen mit der Zivilgesellschaft und Unternehmen zu verknüpfen. Dazu gehören Kooperationsprojekte mit Partnern außerhalb der Hochschullandschaft, Netzwerke und regionale Arbeitskreise, z. B. mit Kommunen oder auch Angebote im Bereich der Weiterbildung.
Spin-offs werden häufig in wissens- und zukunftsorientierten Branchen gegründet. So können in Hochschulnähe zukunftsorientierte Branchengruppen (cluster) und wirtschaftliche Verdichtungen entstehen (Zhan W06: 179).

Die Hochschule kann als zentrale feil der regionalen Wissensinfrastruktur als Inkubator agieren, Gründer unterstützen und ein lebendiges Gründungsklima fördern (Fritsch/Henning/Slavtchev et al. 2007: $161 \mathrm{ff}$. ). Ob und inwieweit die Region von Spin is einer Hochschule profitiert, ist zentral davon abhängig, ob Gründer in der Region verbleiben oder in andere Regionen abwandern. Der regionale Verbleib hängt von persönlichen, ragionsund hochschulseitigen Einflussfaktoren ab. Für str jische Überlegungen einer Hochschule und politischer Entscheidungsträger, inwieweit Mittel für Gründerförderung aufgewendet werden, ist es somit wichtig, die Einflüsse auf den regionalen Verbleib akademischer Gründer zu kennen. Der vorliegende Beitrag untersucht diese Faktoren für akademische Gründungen der Internetbranche. Folgende Schlüsselfragen werden betrachtet: Welcher Anteil akademischer Gründer der Internetbranche verbleibt in der Region und trägt somit zum Wissenstransfer zwischen Hochschule und Region bei? Von welchen Faktoren hängt die Standortwahl im Internetbereich $a b$ ?

\section{Spin-off-Gründungen - ein regionalökonomischer Selbstläufer?}

Gründungen - und besonders innovativen Gründungen gehen langwierige Planungsphasen voraus, in denen Produktkonzepte entwickelt, Grundlagenforschung, Marktund Standortanalysen durchgeführt werden (Frits 74, 96). Die tatsächliche Gründung erfolgt in der $t$ en Start-up-Phase, hier wird das Produkt zur Markt- und Produktionsreife geführt und ein Business- und Marketingkonzept entwickelt. Erst später folgt eine wirtschaftlich orientierte Expansionsphase, mit der Produktionsbeginn und Markteinführung einhergehen. In den frühen Phasen des Gründungsprozesses wird eine der wichtigsten Entscheidungen gefällt: die vorläufige Standortwahl (Fritsch 2016: 96). Sie bestimmt die Konkurrenzsituation, den Zugang zu Ressourcen, Humankapital, Absatzmärkten, Kapitalgebern und Infrastruktur (Schmidt/Heinrichs/Walter 2010: 11). Die Bedeutung einzelner Faktoren ist in der Stando hl kaum pauschal zu betrachten, da sie unter Einfluss der Geschäftsidee, der produkt- und branchenspezifischen Marktbedingungen und den Kundenbedürfnissen variiert. Für viele Spin-off-Gründer ist jedoch die Nähe zur Alma Mater wichtig (Schmidt/Heinrichs/Walter 2010: 10). Besonders die frühen Gründungsphasen sind 2 bit unzureichender wirtschaftlicher Tragfähigkeit und hoher Unsicherheit verbunden. Gründer sind daher auf Unter- 
stützungsnetzwerke und Fördermittel angewiesen, welche Hochschulen bieten oder vermitteln können (Schefczyk 2004: 42; Neubecker 2006: 36 ff.). Gelingt es, Gründer in diesen Phasen an et Region zu binden, ist die Wahrscheinlichkeit eines langfristigen Verbleibs hoch.

Sowohl die Gründungswahrscheinlichkeit als auch die Standortwahl hängen von individuellen personenbezogenen Faktoren ab (Schleinkofer 2006: 63) - der Persönlichkeit, sozialen Netzwerken und anzieller Unterstützung. Nachfrage- oder umfeldorientierten Ansätzen zufolge beeinflussen neben diesen individuellen Faktoren auch Einflüsse des makro- und mikrosozialen Umfeldes die Gründungsneigung (Blum/Leibbrand 2001: 115; Backes-Gellner/Demirer/Sternberg 2,02: 79) esen Ansätzen zufolge filtern potenzielle Grü $\longrightarrow$ die regionalen Umfeldeinflüsse auf der Basis individueller personenbezogener Faktoren (Backes-Gellner/Demirer/Sternberg 2002: 63, 79), was zu unterschiedlicher Gründungsneigund $\mathrm{n}$ Personen einer Region (intraregional) und zu variierender Gründungshäufigkeit verschiedener Regionen (interregional) führt. Die Hochschule stellt einen wichtigen Aspekt des mikrosozialen Umfeldes potenzieller akademischer Gründer dar, zudem kann der mehrjährige Aufenthalt der Absolventen in der Hochschulregion zu einem Verbleib innerhalb der Region beitragen. Die zentralen nicht personenbezogenen Umfeldeinflüsse auf die Standortwahl einer Gründung beziehen sich somit einerseits auf die Alma Mater, andererseits auf die Hochschulregion.

\subsection{Hochschulseitige Faktoren}

Die Gründungsorientierung als strategische Ausrichtung der Alma Mater beeinflusst potenzielle Gründer maßgeblich (Di Gregorio/Shane 2003: 213). Gründungsneigung wird durch Hochschulen ördert, indem gründungsrelevantes Wissen vermittelt sowie Infrastruktur, Beratung und Unterstützung zur Verfügung gestellt werden. Diese „Entrepreneurship-Education“ und der „EntrepreneurshipSupport" können durch die Hochschule bzw. die Region erfolgen oder im Rahmen öffentlicher Förderprogramme wie EXIST $^{2}$ (Hamm/Jäger/Karl et al. 2013a: 74). Zudem rden Neugründungen oftmals vo bchschulnahen Unternehmen (Inkubatoren), Geräte und Räume mit Kommunikationsinfrastruktur zur Verfügung gestellt (Kulicke/ Dornbusch/Kripp et al. 2Q12: 20). Fraglich ist, ob neben der Gründungsneigung au lie Verbleibsquote der Gründer beeinflusst wird.

Ein Einfluss der Hochschulgröße auf die Verbleibswahrscheinlichkeit wird vermutet. Denn große Hochschulen verfügen über bessere technische und räumliche Infrastruktur sowie mehr Ressourcen, um Einrichtungen zur Grün-

${ }^{2}$ Vgl. http://www.exist.de/DE/Home/inhalt.html (15.07.2016). derförderung zu unterhalten (Di Gregorio/Shane 2003). So lohnt sich der Aufbau eines Inkubatorzentrums ve $y$ kt für große Hochschulen, da eine kritische Anzahl von Gründern eher überschritten wird und Synergieeffekte für die Fakultäten zu erwarten sind. So zeigen Landry/Amara/Rherrad (2006), dass sich die Größe kanadischer Hochschulen positiv auf Gründungsneigung Hochschulangehöriger auswirkt. Da gründungsbezogene Hochschulaktivität während und nach dem Gründungsprozess relevant ist, aber eher in Anspruch genommen werden kann, wenn die Gründung in räumlicher Hochschulnähe erfolgt, ist davon auszugehen, dass die Hochschulgröße sich auf die Gründungshäufigkeit und den regionalen Verbleib positiv auswirkt. In Regionen mit großen Hochschulen und einer entsprechend hohen Anzahl potenzieller Gründer ist des Weiteren eher der Umstand gegeben, dass eine kritische Masse neuer Unternehmen gleicher oder ähnlicher Branchen zur Bildung eines Branchenclusters beiträgt, das für die Entstehung und den Verbleib von Gründungen förderlich ist (Iacobucci/Micozzi 2015: 440).

Da akademische Gründungen oft in wissensintensiven, zukunftsorientierten Bereichen erfolgen, dürfte die Forschungsintensität und -qualität der Hochschule die Gründungshäufigkeit der Hochschulakteure beeinflussen. Je intensiver, hochwertiger und marktkonformer Hochschulforschung durchgeführt wird, desto wahrscheinlicher ist eine Ausgründung (Di Gregorio/Shane 2003: 212). Auch hier ist fraglich, ob die Forschungsinter it die Standortwahl beeinflusst. Dies könnte der Fall sein, wenn Spin-offs vermehrt von Hochschulakteuren gegründet werden, die während der unternehmerischen Anfangsphase ihre Anstellung im Bereich der Forschung einer Hochschule behalten und auf räumliche Nähe etwa zur Forschungsinfrastruktur der Hochschule angewiesen sind.

Gründungen unterscheiden sich nach Hochschultyp. Dabei sind vor allem Fachhochschulen und Universitäten zu unterscheiden. Unterschiede zeigen sich in der Häufigkeit, Erfolgswahrscheinlichkeit, fachlichen Ausrichtung, Wissens- und Forschungsintensität (BMWI 2N08: 14; Hamm/ Jäger/Karl et al. 2013a: 66). Studien bldstein 2009; Dinse/Hamm/Jäge al. 2014) zeigen zudem, dass hhochschulen im Vergleic universitäten eine stärker regionale Wissenstransferausrichtung haben - daher wird für Gründungen durch Fachhochschulakteure eine höhere Verbleibsquote erwartet.

\subsection{Regionsseitige Faktoren}

Für viele Gründer ist der Agglomerationsgrad ein wichtiger Standortfaktor, die Standortwahl fällt häufig auf $\mathrm{Ag}$ glomerationskerne (Egeln/Gottschalk/Rammer et al. 2002: 44). Urbane Zentren gelten als vorteilhaft für Gründ da eine gute Infrastruktur und die räumliche Nähe zu Kunden, 
Lieferanten, Dienstleistern und Kooperationspartnern gegeben ist. Zudem entstehen durch die wirtschaftliche Verdichtung Spill-Over-Effekte. Agglomerierte Zentren sind aufgrund hochqualifizierter Arbeitnehmer, auf Gründer ausgerichteter Förderleistungen, einer guten Wissensinfrastruktur sowie Spill-Over diverser Wissenschaftseinrichtungen speziell für wissensintensive Gründungen sinnvoll (Bruns/ Görisch 2002: 13; Heumann 2010: 61; Fritsch/Schroeter 2011: 32

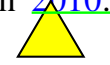

Die wirtschaftlichen Gegebenheiten sind ein weiterer Parameter, der die Standortwahl beeinflussen könnte. Hier gibt es keine eindeutige Aussage in der Fachliteratur. Egeln, Gottschalk und Rammer (2004: 211) zeigen, dass die Standorte der prominentesten In ator-Hochschulen fast durchweg in wirtschaftlich und infrastrukturell attraktiven Regionen liegen. Eine Studie der Absolventenmobilität deutet darauf hin, dass sich eine positive Situation auf dem Arbeitsmarkt negativ auf die Mobilitätsbereitschaft von Akademikern auswirkt (Bruns/Görisch 2002: 37). Laut Möller (2014) zählt eine hohe Wirtschaftsleistung boch nicht zu den Hauptkriterien der Standortwahl. Die Konjunktur bzw. exogene konjunkturelle Schocks beeinflussen nachweislich das Gründergeschehen (Fritsch 2N16: 85), eine konjunkturell bedingte hohe Arbeitslosen kann zu vermehrten sogenannten notwendigen Gründungen (Necessity Entrepreneurship) führen, da die Gründung dann ein mögl $r$ Weg aus der Arbeitslosigkeit ist. Dieser Zusammenhang gilt für wissensintensive Gründungen als umstritten. Zusammengefasst wird ein Zusammenhang der ökonomischen Lage und des Arbeitsmarktes mit der Gründungsaktivität vermutet, dessen Ausprägung a priori nicht eindeutig ist.

$\mathrm{Ob}$ eine Gründung in der Region erfolgt, hängt auch von der regionalen Branchenstruktur ab. Gründungen erfolgen häufiger in urbanen dienstleistungsgeprägten Agglomerationen als in monoindustriellen, primär fertigungsgeprägten Agglomerationen (Audretsch/Falck/Feldman et al. 2012: 387). Darüber hinaus zeigen Egeln, Gottschalk und Rammer (2004) sowie Fritsch und Aamoucke (2013), dass Spin-offs häufiger in der Region des Inkubator bründet werden, wenn dort eine hohe Konzentration von Unternehmen in der Branche des jeweiligen Spin-offs vorliegt.

Ebenso ist die Wissens- und Innovationsorientierung der Wirtschaft ein Faktor der Standortwahl. Akademische Gründungen werden meist den wissens- und innovationsintensiven Branchen zugeordnet, sie profitieren von einer Wirtschaftsstruktur, in der eine hohe Wissensbasis vorherrscht (Fritsch/Aamoucke 2013: 873). Zudem profitieren Gründer von gut vernetzten bovativen, forschungs- und wissensintensiven Branchen, die das Ausbildungs-, Zuliefer- und Dienstleistungsangebot, die Nachfrage wissensintensiver Güter und Dienstleistungen und Kooperationen ermöglichen.

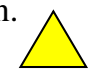

Infrastruktur als wichtiger Standortfaktor beinhaltet unter anderem die Wissensinfrastruktur. Hierzu zählen Einrichtungen. die Wissen und Technologie fördern und von Bedeutung für Gründer sind, da besonders in der Gründungsstartphase die Nähe zum Inkubator oder zu Forschungspartnern wichtig ist (Bruns/Görisch 2002: 14; Goldstein 2005: 206, 219). Die gründungsbezogene Infrastruktur ist von Bedeutung. da gründungsrelevante Unterstützungsangebote auf regionaler Ebene variieren. So werden Beratungs- und Fördermaßnahmen, Stipendien und Gründerwettbewerbe oft individuell durch regionale Akteure durchgeführt. Die Verkehrsinfrastruktur ist ein weiterer Standortfaktor, da die gute Erreichbarkeit einer Region einen positiven Einfluss auf das regionale Gründungsgeschehen nimmt (Fromhold-Eisebith 1992: 60; Bruns/ Görisch 2002: 14: Audretsch/Falck/Feldman et al. 2012: 387). Speziell für Gründer im Internetbereich ist zudem die Verbreitung schneller und hochwertiger Internetzugänge für den eigenen Gebrauch und den der Kunden unerlässlich und eine standortqualitätsbestimmende Komponente.

Auch weiche Standortfaktoren wie Umwelt, Freizeitund Kulturangebot beeinflussen die Standortwahl. Das Gründungs- und Innovationsklima gilt als wichtiger Faktor, da ein innovatives, dynamisches Umfeld Gründungsneigung und -erfolg fördert (Hemer/Walter/Berteit et al. 2006: 19). Netzwerk- und Förderleistungen sowie Aus- und Weiterbildung. Beratung und finanzielle Unterstützung innerhalb eines Netzwerks fördern dieses Gründungsklima (Koschatzky 2002: 31). Nach den Theorien von Florida (2002) zur „Kreativen Klasse“ haben die Kreativen, denen meist akademische Gründer zuzuordnen sind, in Bezug auf ihre Standortwahl klare Präferenzen, die sich vor allem in weichen Standortfaktoren widerspiegeln. Eine hohe Standortqualität aus der Sicht Kreativer zeichnet sich durch ein tolerantes und durch Vielfältigkeit geprägtes Klima aus, in dem sich kulturelle Impulse gegenseitig bereichern. Durch die Vielzahl kreativer Talente entsteht eine innovationsfreudige Stimmung, die Unternehmen aus Bereichen der wissensintensiven Dienstleistungen und Zukunftstechnologien anzieht. Je stärker das kreative Milieu an einem Standort, desto eher eignet sich dieser für die Standortwahl. Ergänzend sei die thesenbehaftete Natur von Floridas Ansätzen betont. eine empirische Kausalität kann nicht vollkommen belegt werden.

\section{Die Gründerszene - empirische Ergebnisse}

\subsection{Der Datensatz}

Für das Vorhaben wird eine individuelle empirische Basis benötigt, die insbesondere Informationen zu Gründungsund Hochschulstandorten sowie ihre geographische Distanz 
und dem akademischen Hintergrund des Gründers erfasst. Mehrere Datenbanken wurden auf ihre Eignung geprüft, unter anderem das „Sozioökonomische Panel“, das „Mannheimer Gründungspanel“, die Gewerbeanzeigenstatistik, die EXIST-Begleitforschung und die KOAB Absolventenstudie (INCHER 2011). Öffentlich zugängliche Daten über den regionalen tand an Gründern aus Hochschulen und die Hintergründe der Standortwahl bestehen bisher nicht flächendeckend, was eine Primärerhebung begründet.

Die Studie basiert auf der Erhebung von Daten einer öffentlich zugänglichen Gründerdatenbank der Internetplattform Gründerszene, ein Online-Produktportfolio der Vertical $\mathrm{MmbH}$, die speziell auf Gründer im weitgefassten Bereich der Internetbranche spezialisiert ist. ${ }^{3}$ Dje Gründer lassen sich nicht einer Branche zuordnen, $Z$ verden Dienstleistungen in den Bereichen Online- und Eventmarketing, Human Resources, Consulting, Social Community, Finanz-, Logistik-, rechtliche und IT-Dienstleister erfasst. Den Gründungen ist eines gemein: Sie nutzen das Internet als Marketing- und Angebotsplattform und als zentralen Distributionskanal und bewegen sich im Bereich der digitalen Dienstleistungswelt. Als Gründung ist hier die Errichtung eines Unternehmens zu verstehen, eine Umbenennung oder Rechtsformänderung wird nicht als Gründung charakterisiert. Aufgrund der Konzentration der Gründerszene speziell auf eher innovative Neugründungen (Start-ups) und Entrepreneure der Internetbranche dürfte ein hoher Anteil der portraitierten Gründungen der von Schumpeter geprägten Diskussion des Entrepreneurship entsprechen. Fritsch (2016: 9) umschreibt dieses innovative Entrepreneurship als I ngsfunktion im Sinne des Unternehmertums in neu gegründeten Unternehmen, die innovative Marktneuerungen einführen. Da der Grad der Wissensintensität, Innovativität und der Geschäftserfolg der Gründungen nicht erhoben werden kann, werden die generierten Datensätze im Rahmen dieser Studie pauschal als Gründungen und Gründer bezeichnet.

Die Datenbank der Gründerszene stellt jeweils das Unternehmen und den Gründer vor - dies umfasst eine Verlinkung zu Profilen in den Netzwerken Xing, Facebook und Linked-In. Im Erhebungszeitraum in 2013 umfasste die Datenbank deutschlandweit 3436 Gründer. Da nicht für alle Gründer ausreichend Daten extrahiert werden konnten, wurden 1685 Gründungen analysiert. Ein Großteil persönlicher Charakteristika als Erklärungsvariable der Standortwahl ist der Datenbank nicht zu entnehmen und kann nicht berücksichtigt werden. Die für die Studie maßgebliche Information besteht in der Verbindung zwischen dem Gründer, dessen letzter Alma Mater und seiner Gründung. Dies ermöglicht eine Bestimmung der Distanz zwischen Gründung und Alma Mater (bei Hochschulen mit mehreren

\footnotetext{
${ }_{3}$ http://www.gruenderszene.de/ (16.07.2016).
}

Standorten gilt der Hauptstandort als Berechnungsgrundlage). Somit kann der durch die Gründung generierte Wissenstransfer auf eine Hochschule zurückgeführt und dem geographischen Aspekt der Transferreichweite Rechnung getragen werden.

Die Interpretation der erzielten Resultate findet vor dem Hintergrund einiger Erhebungs- und Datenbankprobleme statt. Zum einen ist unklar, wie repräsentativ das Gründergeschehen akademischer Internetgründer in Deutschland widergespiegelt wird. Die Erfassung von Gründern erfolgt, indem sich diese in die Datenbank einschreiben, zudem werden Gründer aufgenommen, über die Artikel veröffentlicht werden und die an Veranstaltungen der Gründerszene teilnehmen. Die Stichprobe dürfte mithin aus Gründen der Selbstselektion verzerrt sein, sodass sich die Gründer in attitudinalen und strukturellen Merkmalen der Gründung von der Grundgesamtheit unterscheiden. So ist davon auszugehen, dass erfolgreiche Gründer eher dazu neigen, sich in einer reellen und digitalen „Gründungs-Community“ $\mathrm{zu}$ vernetzen, wobei erfolgreiches Networking als kausaler Erfolgsfaktor einzuschätzen ist (Krebs 1895: 114).

Auch eine geographische Verzer g ist zu erwarten, da Berlin als Hotspot der deutschen Internetbranche gilt und Vertical Media hier lokalisiert ist, daher finden initiierte Events in der Hauptstadt statt. Tatsächlich liegen 651 der 1685 betrachteten Gründungen in Berlin. Es ist nicht auszuschließen, dass aufgrund persönlicher Kontakte zwischen Plattformbetreibern und Gründern eine überproportionale Repräsentanz besteht. Ein gesonderter Schätzansatz ohne Gründungen in Berlin liefert jedoch keine deutlich abweichenden Ergebnisse (vgl. Anhang). Eine weitere Verzerrung kann durch die Häufigkeit einzelner Hochschulen im Datensatz entstehen, die besonders viele Gründer hervorbringen, z. B. aufgrund eines gründungsaffinen Curriculums. Die Folge sind Gruppen betrachteter Merkmalsträger, die gleiche Hochschulkennzahlen aufweisen, wodurch eine Verzerrung der Koeffizienten und der Schätzung der Standardfehler auftreten kann. Um das Modell robuster $\mathrm{zu}$ machen, wird ein spezieller 2D-Clusteransatz mit adjustierten Standardfehlern genutzt (Petersen 2009). Zudem stellt der genutzte Datensatz nicht zweif rei fest, ob eine Gründung im Sinne eines Forschungs-Spin-offs in Zusammenhang mit der Hochschule steht. Wäre dies möglich, könnten hochschulseitige Einflüsse deutlicher herausgestellt werden.

Der Beitrag bezieht sich auf Gründungen einer Branche. Eine Übertragung der Ergebnisse auf andere Branchen ist aufgrund der spezifischen Eigenschaften der Internetbranche bedingt möglich. Für den Forschungszweck eignet sich diese Branche durch hohe Gründungsaktivität und geringe Markteintrittskosten. Zudem werden für Produktinnovationen nicht hohe finanzielle Investitionen und langlebige Entwicklungszyklen benötigt. Somit werden Innovatio- 
Tab. 1 Rankings der Gründungs- und Alma-Mater-Standorte

\begin{tabular}{|c|c|c|c|c|c|c|c|c|}
\hline \multicolumn{5}{|c|}{ Gründungsort } & \multicolumn{4}{|c|}{ Standort der Alma Mater } \\
\hline \multicolumn{3}{|c|}{ Ranking - Anzahl Gründungen } & \multicolumn{2}{|c|}{$\begin{array}{l}\text { Bezug zur Bevölkerungsgröße } \\
\text { in } 1000 \text { Einwohner }\end{array}$} & \multicolumn{2}{|c|}{$\begin{array}{l}\text { Ranking - Anzahl Gründer, die } \\
\text { dort studierten }\end{array}$} & \multicolumn{2}{|c|}{$\begin{array}{l}\text { Bezug zur Bevölkerungsgröße } \\
\text { in } 1000 \text { Einwohner }\end{array}$} \\
\hline$\underline{1}$ & Berlin & $\underline{651}$ & Berlin & $\underline{0,19}$ & Berlin & 214 & Mayen-Koblenz & $\underline{0.59}$ \\
\hline$\underline{2}$ & Hamburg & $\underline{190}$ & München & $\underline{0.16}$ & München & $\underline{127}$ & Wiesbaden & $\underline{0,19}$ \\
\hline$\underline{3}$ & München & 167 & Köln & $\underline{0,11}$ & Mayen-Koblenz & $\underline{124}$ & Potsdam & $\underline{0,19}$ \\
\hline$\underline{4}$ & $\underline{\text { Köln }}$ & 111 & Hamburg & $\underline{0,11}$ & Hamburg & $\underline{109}$ & Heidelberg & $\underline{0,17}$ \\
\hline$\underline{5}$ & Karlsruhe & $\underline{25}$ & Karlsruhe & $\underline{0,09}$ & Köln & $\underline{63}$ & Darmstadt & $\underline{0.17}$ \\
\hline$\underline{6}$ & Leipzig & $\underline{25}$ & Jena & $\underline{0,07}$ & Leipzig & $\underline{49}$ & Mannheim & $\underline{0,15}$ \\
\hline$\underline{7}$ & Düsseldorf & 24 & Heidelberg & $\underline{0,07}$ & Karlsruhe & $\underline{43}$ & $\underline{\text { Karlsruhe }}$ & $\underline{0,14}$ \\
\hline$\underline{8}$ & $\begin{array}{l}\text { Frankfurt am } \\
\text { Main }\end{array}$ & $\underline{20}$ & $\underline{\text { Potsdam }}$ & $\underline{0,05}$ & Mannheim & $\underline{36}$ & $\underline{\text { Koblenz }}$ & $\underline{0,13}$ \\
\hline$\underline{9}$ & $\underline{\text { Stuttgart }}$ & 12 & Darmstadt & $\underline{0,05}$ & Wiesbaden & $\underline{34}$ & Würzburg & $\underline{0,11}$ \\
\hline$\underline{10}$ & Heidelberg & $\underline{10}$ & Leipzig & $\underline{0,05}$ & Potsdam & $\underline{28}$ & Erlangen & $\underline{0.10}$ \\
\hline
\end{tabular}

Gesamtdatensatz N = 1685; für 342 Gründungen konnte die Information nicht ermittelt werden

nen nicht nur durch große Institutionen erwirkt, vielmehr gibt es eine hohe Fallzahl individueller Entrepreneure, oft mit akademischem Hintergrund. Für die Bestimmung der Unterschiede verschiedener Hochschultypen ist die Internetbranche geeignet, da die Fächergruppen, aus denen die Gründungen erfolgen, in ähnlichem Umfang an Universitäten und Fachhochschulen gelehrt werden. ${ }^{4}$ Zydem können im Verbleib der Gründer keine systematisch Cäumlichen Verzerrungen auftreten, wie sie durch bundeslandweit aufgeteilte Lehramtsstudierende entstehen könnten.

\subsection{Deskriptive Statistik}

Der erhobene Gesamtdatensatz enthält 1685 Gründungen von 1067 Gründern. Die Geschlechterverteilung liegt bei $94,7 \%$ männlichen und 5,3\% weiblichen Gründern. Mehr als die Hälfte der Firmen, und zwar 998, wurden von Universitätsabsolventen gegründet, gefolgt von 334 Gründungen durch Fachhochschulabsolventen, 242 Gründungen durch Absolventen Technischer Universitäten sowie 20 Gründungen der Kunsthochschulabsolventen (91 Gründer gaben keine Hochschultypzugehörigkeit an). Um die Gründungsneigung nach Hochschultyp zu vergleichen, wurde die Zahl der untersuchten Gründungen mit der Anzahl der eingeschriebenen Studierenden ins Verhältnis gesetzt. ${ }^{5}$ Für Universitäten ergibt sich ein Gründungsquotient von $\$$ untersuchten Gründungen pro 1000 eingeschriebenen Studierenden, für Technische Universitäten ein Quotient von 0,83 und für Fachhochschulen 0,49. Absolventen von Universitäten und Technischen Universi-

\footnotetext{
${ }^{4}$ Berechnungen auf der Datenbasis: Statistisches Bundesamt (2013).

$\underline{5}$ Die Zahl der Gründungen nach Hochschultyp wird durch die Zahl eingeschriebener Studierender (in 1000) dieses Hochschultyps in 2011 geteilt: Datenbasis: Statistisches Bundesamt (2013).
}

täten der betrachteten Stichprobe gründen deutlich häufiger als Fachhochschulabsolventen.

Tab. 1 zeigt eine Bewertung der Gründungs- und AlmaMater-Standorte der Stichprobe (sample). Der beliebteste Gründungsort ist Berlin mit $651 \mathrm{G}$ Jungen. Auf den weiteren Plätzen liegen die Großstädte Hamburg (190), München (166) und Köln (111). Auch in der Betrachtung, die die Anzahl der Gründungen in Bezug zur Einwohnerzahl der Standorte setzt, liegen metropolitane Großstädte auf den ersten Plätzen. Die rechte Seite der Tabelle zeigt ein Ranking der Alma-Mater-Standorte. 214 der untersuchten Gründer studierten in Berlin. Demnach attrahierte Berlin im Saldo über 400 Gründer. Überraschend viele Gründer haben in Mayen-Koblenz studiert - dort bietet die „WHU Otto Beisheim School of Management" einen speziell auf Gründer ausgelegten Studiengang an.

Abb. 1 zeigt, dass 40,3\% der unter hten Gründungen innerhalb von $50 \mathrm{~km}$ um die Hochschule stattfinden. Absolventen aus Fachhochschulen und Technischen Universitäten gründen deutlich häufiger am Alma-Mater-Standort. Auffällig sind vor allem die hohen regionalen Gründungsraten der Technischen Hochschulen. 53,0\% der Gründungen durch Absolventen Technischer Universitäten erfolgen innerhalb eines 50-km-Radius um die Hochschule, für Fachhochschulen liegt dieser Wert bei $45,5 \%$, für Universitäten bei $34,5 \%$. Werden Universitäten und Technische Universitäten nicht differenziert, erfolgen $38,7 \%$ der Gründungen durch Universitätsabsolventen im 50-km-Umkreis. Der vergleichsweise stärkere Regionalbezug der Gründungen durch Fachhochschulabsolventen ist vor dem Hintergrund interessant, dass lediglich in der Betrachtung ländlicher Räume Fachhochschulen im Vergleich zu Universitäten über ein stärker regionales Einzugsgebiet verfügen. Gleichzeitig gilt die Größe des Einzugsgebietes einer Hochschule als wichtige Erklärungsdeterminante der potenziellen Mi- 
Abb. 1 Entfernung zwischen Hochschule und Gründungsort
Abb. 2 Anzahl an Gründungen und Anteil regionaler Gründungen nach zeitlichem Abstand zum Studienende. Gesamtdatensatz $N=1685$; für 283 Gründungen konnte die Information nicht ermittelt werden
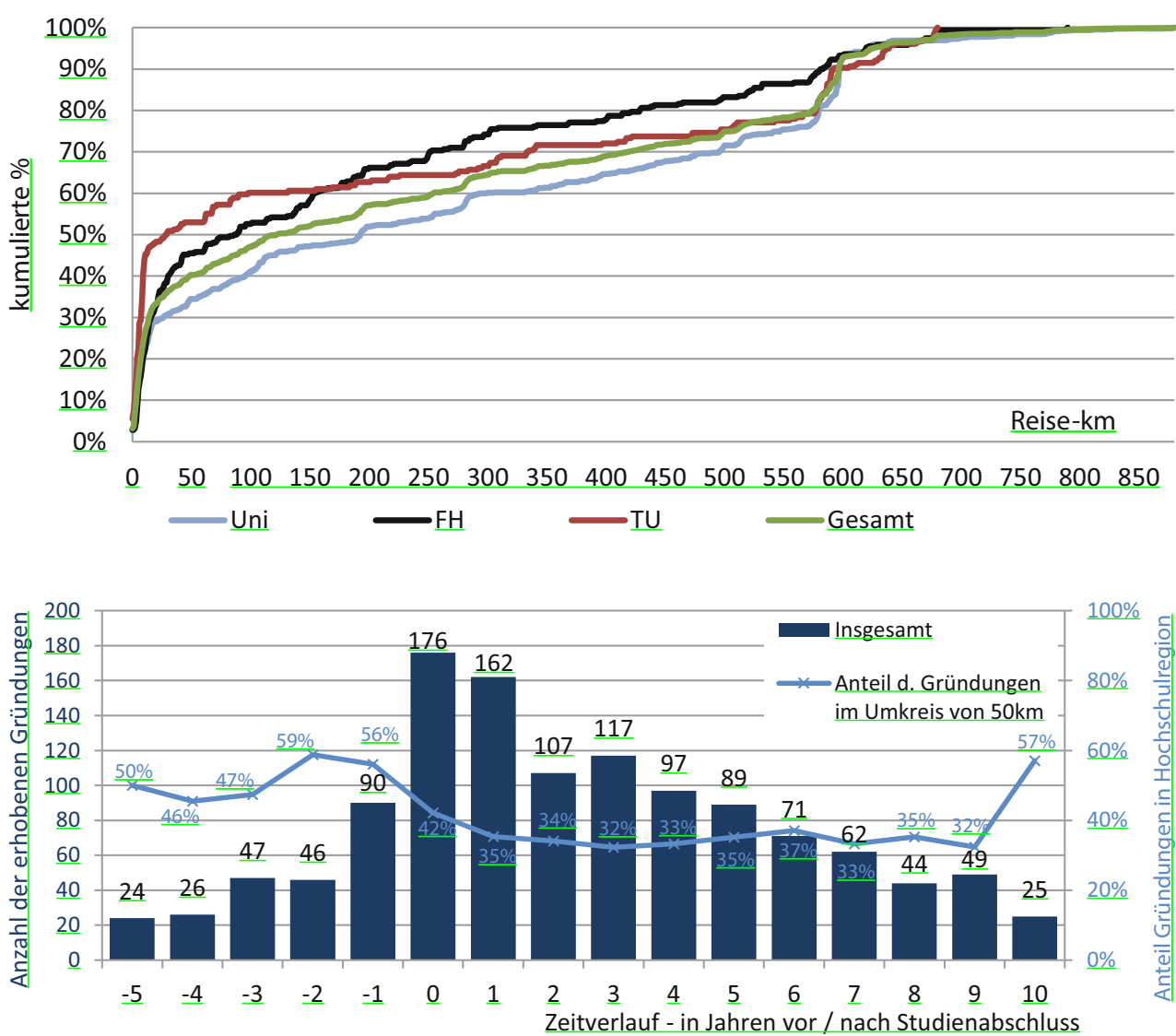

gration von Hochschulabsolventen (Kratz/Lenz 2015: 15). Während Gründungen durch Fachhochschulabso ten anteilsmäßig häufiger in der Region ihrer Fachhochschule erfolgen, ist festzuhalten, dass Universitäten absolut - durch höhere Absolventenzahlen und Gründungsquote - deutlich mehr regionale Gründungen generieren.

Eine weitere Auffälligkeit wird bei 600 Kilometern Entfernung deutlich, wo die Kurven der Universitäten einen steilen Anstieg verzeichnen. Begründet ist dies wahrscheinlich in der Entfernung zwischen beliebten Hochschulorten in Nordrhein-Westfalen wie Köln oder Düsseldorf, und dem beliebtesten Hochschulstandort Berlin.

Die meisten Gründungen des Samples erfolgen unmittelbar nach Studienende sowie mit einem Jahr Verzögerung (vgl. Abb. 2ג. Diese Fälle machen 27,4\% der Gründungen zwischen $\mathrm{Jahre}$ vor und zehn Jahre nach dem Studienende aus. Je länger der Abschluss zurückliegt, desto seltener erfolgt eine Gründung. Zudem erfolgt bereits während des Studiums eine relevante Zahl an Gründungen. Der zeitliche Abstand einer Gründung zum Abschluss hat offenbar keine zwingende Auswirkung auf die Entscheidung, ob in der Nähe der Alma Mater gegründet wird. Deutlich wird allenfalls, dass Gründungen kurz vor oder zum Abschluss sich vergleichsweise häufiger innerhalb des 50-km-Radius um die Alma Mater ansiedeln.

\subsection{Regressionsanalyse - Methodik}

Die Studie analysiert die Standortwahl akademischer Gründer aus Hochschulen in Deutschland und ihre regions- und hochschulseitigen Determinanten. Dafür wird mit einer Logit-Schätzung untersucht, welche Faktoren die Wahrscheinlichkeit mitbestimmen, dass eine Gründung innerhalb der Region erfolgt. Die binäre abhängige Variable ist mit den Ausprägungen 1 (Gründung in der Hochschulregion) und 0 (Gründung außerhalb) definiert. Der Logit-Ansatz wurde gewählt, da aus regionalökonomischer und hochschulpolitischer Sicht speziell der regionale Verbleib der Gründer im Sinne einer grundsätzlichen Entscheidung wichtig ist. Wählen sie einen anderen Standort, ist es für die Region zunächst unerheblich, wie weit dieser exakt entfernt ist.

Bei Studien zu regionalökonomischen Hochschuleffekten besteht grundsätzlich die Frage der Abgrenzung der Hochschulregion. Die räumliche Differenzierung ist eine Herausforderung, deren methodische Operationalisierung auf der Basis von Praktikabilität, Datenverfügbarkeit und theoriebezogener Fundierung entschieden wird. Für eine detaillierte Diskussion wird auf Hamm, Jäger, Karl et al. (2013b: 109) verwiesen. Gängige Analyseeinheiten sind Städte, K e, Reisedistanz und Reisezeit, Regierungsbezirke, Postleitzahl-, Raumordnungs- und Arbeitsmarktregionen (Beyer/Majer 2002). Wie groß eine Hochschulregi- 
on tatsächlich ist, dürfte sich nach Hochschultyp, Zentralität der Region und Agglomerationsstufe unterscheiden. Inhaltlich wird der Thematik im personenbezogenen Wissenstransfer eine räumliche Abgrenzung abseits administrativer Grenzen im Rahmen einer Reisedistanzanalyse besser gerecht. Sobald Individuen oder persönlicher, interagierender Kontakt Transferprämissen sind, sollte eine Hochschulregion einen Radius von ca. einer Stunde Pendeldistanz nicht überschreiten, da darüber hinaus die Wissensübertragung zunehmend erschwert wird. Im vorliegenden Ansatz wird von einer regionalen Gründung gesprochen, wenn diese innerhalb eines 50-km-Raiseradius ${ }^{6}$ um den Hauptstandort der Alma Mater erfolg Vilometrische Reisedistanzen geben einen guten Ansatzpunkt, aber keine vollständige Information über den Zeitaufwand, der notwendig ist, um diese Distanz zurückzulegen. Da die Abgrenzung der Hochschulregion mittels eines Schwellenwertes nicht unproblematisch ist, wurde die Berechnung um ein Verfahren der kleinsten Quadrate (ordinary least sauares, OLS) ergänzt, das die Distanz zwischen Alma M und Gründungsort in $\mathrm{km}$ als abhängige, lineare Variable betrachtet.

Die Studie ist auf hochschul- und regionsseitige Einflussfaktoren konzentriert. Sicherlich fließen in die Standortentscheidung der Gründer soziale, personenbezogene und individuelle Faktoren mit ein, die aufgrund fehlender Datenverfügbarkeit nur sehr begrenzt dargestellt werden können. Einzig die Jahre zwischen Abschluss und Gründung konnten als parsonenbezogene Variable in ausreichender Qualität erhobe lerden.

Das Logit-Schätzmodell wird in drei Versionen gerechnet. Modell 1 berücksichtigt alle Gründungen im Datensatz zwischen 2007 und 2014 durch Gründer mit akademischem Abschluss. Die besondere Situation akademischer Spin-offGründer wird insofern betrachtet, als das Modell 2 nur Gründungen innerhalb von zwei Jahren vor bis fünf Jahre nach Studienabschluss des Gründers enthält. Modell 3 umfasst ausschließlich Gründungen außerhalb dieses Zeitraums. Der Vergleich soll Aufschluss darüber geben, ob Spin-off-Gründer und sonstige akademische Gründer in ihrer Standortwahl unterschiedlich auf regionale und hochschulseitige Einflussfaktoren reagieren.

Auf der Basis der beschriebenen Faktoren der Standortwahl akademischer Gründer wurden unter Berücksichtigung der Datenverfügbarkeit sowie deren Abbildungsstärke Indikatoren ausgewählt, deren Einfluss im Regressionsmodell geprüft wird. Dem nachfrage- und umfeldorientierten Ansatz zufolge beeinflussen hochschulseitige Faktoren die

${ }^{6}$ Ein Excel-Hilfsprogramm (Add-on) berechnet die Distanz zwischen den Mittelpunkten der Pastleitzahlgebiete. Grundlag t der motorisierte Individualverkehr ffgrund der Verkehrssituation in M bolen wäre in weiteren Studien eine Ergänzung des öffentlichen Nahverkehrs interessant.
Standortwahl. Um die strategische Gründungsorientierung einer Hochschule empirisch abzubilden, stellt eine DummyVariable ihre eventuelle Aktivität im Rahmen der EXISTFörderung dar (Kulicke/Dornbusch/Kripp et al. 20 \22). Als Maßzahl für die Hochschulgröße wird die $\mathrm{Z}$ Studierender in 1000 der Alma Mater, als Indikator für die Forschungsintensität und -qualität die Drittmittelhöhe je Professor in das Modell aufgenommen. Eine Dummy-Variable unterscheidet zudem Gründer von Universitäten und Fachhochschulen.

Auch regionsseitige Faktoren der Hochschulregion beeinflussen die Standortwahl. Der Indikator Bevölkerungsdichte (Einwohner pro $\mathrm{km}^{2}$ ) bildet den Agglomerationsgrad ab. Die Bruttowertschöpfung je Einwohner gibt Aufschluss über die größenbereinigte Wirtschaftsleistung, dia Beschäftigungsquote betrachtet Chancen am Arbeits Um erfassen zu können, inwieweit die vorliegende Branchenstruktur den untersuchten Gründungen entspricht, wurden die Anteile Erwerbstätiger in der Dienstleistungs- und der Internetbranche ${ }^{7}$ analysiert. Der erste Indikator besch die Passgenauigkeit im weiteren, letzterer die Passgenauigkeit im engeren Sinne. Die Wissens- und Innovationsorientierung der Region wird durch die Variablen „Anteil sozialversicherungspflichtig Beschäftigter in Forschung und Entwicklung“ und „Anteil hochqualifizierter Beschäftigter" abgebildet. Zudem dient die Anzahl wissenschaftlicher Institutionen im Kreis als Proxy-Variable für den Ausprägungsgrad der Wissensinfrastruktur. Für die Qualität der Verkehrsanbindung wird der Indikator „Entfernung des nächsten Autobahnanschlusses in Minuten“ integriert. Ein Indexwert „Kreative Klasse“ $\underline{8}$ jndiziert die Standortattraktivität und ermöglicht eine qual ve Bewertung aus der Sicht Kreativer. Das Gründungsklima wird durch quantitative Indikatoren abgebildet. Ein hoher Anteil Selbstständiger und ein hoher Anteil an Kleinstbetrieben deuten auf ein Klima, das attraktiv für Gründungen ist. ${ }^{9}$

\footnotetext{
7 Zur Analyse des Anteils Erwerbstätiger der Internetbranche wird die Definition des Bundeswirtschaftsministeriums (BMWI 2012) genutzt. Datengrundlage: Sonderauswertung der Bundesagentur für Arbeit 2015, WZ 2008.

${ }_{8}$ Der Indexwert wurde auf der Basis von Anteilswerten der .,Kreativen Klassen“ an allen Erwerbstätigen erhoben; die Messgrößen wurden normiert. Für Details vgl. Gottschalk/Hamm/Imöhl (2010: 109).

${ }^{2}$ Vor der Regression wurden zur Sicherstellung der Abwesenheit räumlicher Korrelation räumliche Korrelationskoeffizienten (Moran's I, Queen contiguity, erster Ordnung) berechnet. Weder die Globalstatistik noch die lokalen Statistiken der Regionen, in denen ein Schwerpunkt der Observation liegt, weisen hohe Korrelationskoeffizienten auf $(0-0,5)$. Die Irrtumswahrscheinlichkeit liegt für alle Variablen unter $5 \%$. Ausnahmen bilden die Variablen „FuE-Beschäftigte“ und „Anteil Kleinstbetriebe“. Da schon die deskriptiven Statistiken keine Hinweise auf räumliche Autokorrelationen geben, wurde auf die Berücksichtigung durch räumliche Regressionsmodelle verzichtet.
} 
Tab. 2 Einflussfaktoren auf die Standortwahl

\begin{tabular}{|c|c|c|c|c|c|}
\hline & Bereich & Indikator & Bezugsjahr & $\begin{array}{l}\text { Erwartetes } \\
\text { Vorzeichen }\end{array}$ & Quelle \\
\hline \multicolumn{6}{|c|}{ Hochschulseitige Faktoren } \\
\hline \multirow[t]{2}{*}{$\begin{array}{l}\text { Strategische } \\
\text { Ausrichtung }\end{array}$} & Gründungsorientierung & EXIST-Förderung-Dummy & $1998-2011$ & + & $\begin{array}{l}\text { Kulicke/ } \\
\text { Dornbusch/ } \\
\text { Kripp } \\
\text { et al. } \\
(2012)\end{array}$ \\
\hline & $\begin{array}{l}\text { Forschungsintensität und } \\
\text {-qualität }\end{array}$ & Drittmittel je Professor & 2007 & + & DeStatis \\
\hline \multicolumn{2}{|c|}{ Hochschulgröße } & Anzahl Studenten in 1000 & 2007 & + & \\
\hline \multicolumn{2}{|c|}{ Hochschultyp } & Dymmy Universität (0)/Fachhochschule (1) & 2007 & + & \\
\hline \multicolumn{6}{|c|}{ Regionsseitige Faktoren } \\
\hline \multirow{5}{*}{$\begin{array}{l}\text { Bevölkerung } \\
\text { Wirtschaft }\end{array}$} & Agglomerationsgrad & Einwohner pro $\mathrm{km}^{2}$ & 2007 & + & BBSR \\
\hline & Wirtschaftsleistung & Bruttowertschöpfung pro Einwohner & 2007 & + & INKAR \\
\hline & Branchenstruktur & $\begin{array}{l}\text { Anteil sozialversicherungspflichtig Beschäftigter in } \\
\text { Dienstleistungsbranchen } \\
\text { Anteil sozialversicherung jichtig Beschäftigter in } \\
\text { der Internetbranche }\end{array}$ & 2007 & \pm & $\frac{\text { INKAR }}{\text { BAfA }}$ \\
\hline & $\begin{array}{l}\text { Wissens-/ } \\
\text { Innovationsorientierung }\end{array}$ & $\begin{array}{l}\text { Anteil sozialversicherungspflichtig Beschäftigter in } \\
\text { Forschung und Entwicklung (FuE) } \\
\text { Anteil sozialversicherungspflichtig } \\
\text { mit Studienabschluss }\end{array}$ & $\underline{2007}$ & \pm & INKAR \\
\hline & Arbeitsmarkt & Beschäftigungsquote & 2007 & + & \\
\hline \multirow[t]{2}{*}{ Infrastruktur } & Wissensinfrastruktur & Anzahl wissenschaftlicher Institutionen im Kreis & 2014 & + & BMBF \\
\hline & Verkehrsinfrastruktur & Fahrtzeit zum nächsten Autobahnanschluss in min & 2012 & - & BBSR $\angle$ \\
\hline \multirow[t]{2}{*}{$\begin{array}{l}\text { Weiche } \\
\text { Faktoren }\end{array}$} & $\begin{array}{l}\text { Gründungs- und Innovati- } \\
\text { onsklima }\end{array}$ & $\begin{array}{l}\text { Anteil Selbstständige } \\
\text { Anteil Kleinstbetriebe }<10 \text { Beschäftigte }\end{array}$ & 2007 & + & INKAR \\
\hline & $\begin{array}{l}\text { Standortqualität aus der } \\
\text { Sicht Kreativer }\end{array}$ & $\begin{array}{l}\text { Indexwert Kreative Klasse } \\
100=\text { bester Indexwert, } 0=\text { niedrigster Wert }\end{array}$ & 2011 & + & NIERS \\
\hline
\end{tabular}

DeStatis Statistisches Bundesamt, BBSR Bundesinstitut für Bau-, Stadt- und Raumforschung, INKAR Indikatoren und Karten zur Raum- und Stadtentwicklung des BBSR, BAfA Bundesamt für Wirtschaft und Ausfuhrkontrolle, BMBF Bundesministerium für Bildung und Forschung, NIERS Niederrhein-Institut für Regional- und Strukturforschung

Tab. 2 zeigt eine Übersicht der regions- und hochschulseitigen Faktoren und die zur Darstellung genutzten Indikatoren. Grundsätzlich sei darauf hingewiesen, dass die Indikatoren die Einflussfaktoren nur indizieren, nicht jedoch in allen Facetten abbilden. Für die Regression wird ein reduzierter Datensatz $N=1336$ genutzt, in dem nur Gründungen zwischen 2007 und 2014 berücksichtigt werden, damit die Indikatoren als Querschnittsvariable mit dem Bezugsjahr 2007 verwendet werden können. ${ }^{10}$

Bei der Vielzahl regionsseitiger Variablen ist nicht auszuschließen, dass Variablen zum Teil gleiche Sachverhalte messen. Eine Korrelationsanalyse der in Tab. 2 angeführten Indikatoren zeigte problematische Korrelationen im Bereich der regionsseitigen Variablen bzw. in der Korrelation einer hochschul- und einer regionsseitigen Variablen (s. Tab. 4 Zusatzmaterial online). Um Multikollinearität zu vermei-

10 Standen Werte für 2007 nicht zur Verfügung, wurde der nächstaktuellere Wert genutzt. Dies ist der Fall bei ..Zahl wissenschaftlicher Institutionen“ und ..Erreichbarkeit nächster Autobahnanschluss“. den, fasst eine explorative Faktorenanalyse Gruppen hoch korrelierender Variablen zu Faktoren zusammen (Backhaus/ Erichson/Plinke et al. 2011: 329). Mithilfe der Faktorenanalyse wurden zwei Fak $\mathrm{n}$ herausgebildet. Daraufhin wurde mit einer VARIMAX-Rotation rotiert, sodass die Indikatoren jeweils auf einem Faktor eine möglichst hohe Ladung und auf dem anderen Faktor eine möglichst geringe Ladung zeigen (Backhaus/Erichson/Plinke et al. 2011: 330). Die beiden Faktoren erklären zusammen $88, \sqrt{ }$ der Gesamtvarianz der regionsseitigen Indikatoren. Faktor 1 „Arbeitsmarkt" umschreibt einen modernen, attraktiven und erfolgreichen Arbeitsstandort und fasst die Variablen Dienstleistungsquote, Einwohnerdichte, Bruttoinlandsprodukt je Beschäftigten, Quote Hochqualifizierter und Index Kreative Klasse zusammen. Es wird erwartet, dass ein wissens- und dienstleistungsintensiver, kreativer, agglomerierter und wirtschaftlich prosperierender Arbeitsmarkt förderlich für die Verbleibsquote der Gründer ist, was ein positives Vorzeichen im Logit-Ansatz impliziert. Faktor 1 „Arbeitsmarkt" erklärt $60,6 \%$ der Gesamtvarianz der ragionsseiti- 
gen Variablen. Der zweite Faktor wird mit „Gründungsklima“ umschrieben, fasst die Quoten der Selbstständigen, Kleinstbetriebe und Beschäftigten zusammen und erklärt weitere $27,5 \%$ der Varianz der regionsseitigen Variablen. Da Regionen mit hohem Anteil Beschäftigter, Selbstständiger und Kleinstbetriebe eine hohe Verbleibsquote der Gründer aufweisen werden, wird im Logit-Ansatz ein positives Vorzeichen erwartet.

Die Variablen ,wissenschaftliche Institutionen“, „Beschäftigtenquote digitale Wirtschaft“ und „Beschäftigtenquote in Forschung und Entwicklung“ konnten aufgrund niedriger Faktorladung keinem Faktor eindeutig zugerechnet werden und führen durch hohe Korrelationswerte indiziert zu Multikollinearität. Daher wurden sie aus dem Modell entfernt. Die Variable „Verkehrsinfrastruktur" konnte keinem Faktor zugeordnet werden, wurde aber aufgrund inhaltlicher Abgrenzung und vergleichsweise niedriger Korrelation mit anderen unabhängigen Variablen als einzelne unabhängige Variable integriert.

Um die Reliabilität bzw. Irrtumskomponente der gebildeten Faktoren abzuschätzen, wird die Methode der internen Konsistenz angewandt. Dieser Schritt wird durchgeführt, um Variablen, die stärker als andere durch eine Irrtumskomponente beeinflusst sind, aus dem Faktor zu entfernen. Zur Messung der internen Konsistenz wird Cronbachs $\underline{\alpha}$ geputzt, dessen Wert abhängig von der Zahl der Varia1 eines Faktors sowie der durchschnittlichen Korrelation der Variablen des Faktors ist (Schmitt 1996). Letztendlich wurden aus dem Variablenset des F Prs 1 „Arbeitsstandort" die Variablen Einwohnerdichte und Kreative Klasse entfernt, aus dem Faktor 2 „Gründungsklima“ die Variable Beschäftigtenquote. Für die gebildeten Faktoren wurde jeweils ein Faktorwert ermittelt, der die einzelnen zu dem Faktor gehörenden Variablenwerte zusammenfasst. Zur Vermeidung von Verzerrungen durch Skalierungsunterschiede wurden bei der Aggregation die Indikatoren standardisiert. Durch das Vorgehen wurde eine Reduzierung auf folgende unabhängige Variablen vorgenommen:

- personenbezogen: Jahre zwischen Studienende und Gründung,

- hochschulseitig: Hochschultyp, Forschungs- und Entwicklungsintensität, Hochschulgröße, EXIST-Förderung,

- regionsseitig: Infrastruktur, Faktor 1 „Arbeitsmarkt“ (Dienstleistungsquote, Bruttoinlandprodukt je Beschäftigten, Quote Hochqualifizierter), Faktor 2 „Gründungsklima“ (Quote Selbstständige und Kleinstbetriebe).

\subsection{Ergebnisse der Schätzungen}

Tab. 3 zeigt die Ergebnisse dreier zentraler Schätzmodelle. Die abhängige Variable der Logit-Schätzung ist eine bi- näre Dummy-Variable, die den Wert 1 annimmt, wenn der Gründer innerhalb einer 50-km-Distanz zur Alma Mater gründet. Modell 1 betrachtet $N=850$ Gründungen unabhängig von deren zeitlichem Abstand zum Abschluss. Modell 2 betrachtet ausschließlich die 557 Gründungen der Datenbank, die innerhalb von zwei Jahren vor bis fünf Jahre nach Studienende stattfanden - hier ist ein inhaltlicher Zusammenhang zum Studium wahrscheinlich, diese Fälle werden hier als Spin-offs bezeichnet. Modell 3 bezieht sich auf akademische Gründer, die außerhalb dieses Zeitraumes gründen. Ein zusätzliches Modell mit Ausschluss der Gründungen in Berlin und eine unterstützende OLS-Schätzung führen nicht zu zusätzlichen Erkenntnissen (vgl. Anhang). Die OLS-Schätzung untersucht als abhängige Variable die Distanz zwischen Hochschule und Gründungsort.

Die Ergebnisse der Modelle ähneln sich grun es sind eils die Variablen Hochschultyp, HochschulgröBe und Gründungsklima signifikant. Aufgrund der unterschiedlichen Skalen der Variablen werden im Folgenden nur die Vorzeichen der Koeffizienten interpretiert, nicht deren Ausprägung. Das Bestimmtheitsmaß liegt bei Pseudo$\mathrm{R}^{2}$ von 0,21 (Modell 1), 0,18 (Modell 2) und 0,25 (Modell 3). Interessant ist im Vergleich der Modelle 1 und 2, dass durch die Hinzunahme der Variablen „Jahre Abschluss/Gründung" und der entsprechenden Spezifizierung der Stichprobe in Modell 1 keine wesentlichen Veränderungen der Werte bei den übrigen Variablen entstehen. Die Entscheidungsfaktoren der Gründer, die kurz vor oder nach Studienende gründen, unterscheiden sich also kaum von den Determinanten der Gründer, die deutlich vor oder nach dem Abschluss gründen. Für sonstige akademische Gründer, die deutlich vor bzw. nach ihrem Abschluss gründen (Modell 3), ist lediglich die Variable Forschungsintensität zusätzlich signifikant.

Die lineare Variable „Jahre Abschluss/Gründung“ gibt die Jahre zwischen Gründung und Abschluss an. Abb. 2 zeigt, dass Gründer mit einer höheren Wahrscheinlichkeit in der Region bleiben, wenn sie vor dem Abschluss gründen. Dieser Zusammenhang liegt nahe, da bei zeitgleicher Gründung zum Studium die räumliche Distanz die zurückzulegende Pendeldistanz ist. Modell 1 bestätigt diese Erkenntnis. Die Eintrittswahrscheinlichkeit einer regionalen Gründung ist erhöht, je niedriger die Jahreszahl zwischen Gründung und Abschluss ist (negatives Vorzeichen). Gründet der Absolvent vor Studienende, bleibt er eher in der Region, im weiteren Verlauf steigt die Abwanderungswahrscheinlichkeit sukzessive an.

Der Hochschultyp ist in allen Modellen als signifikanter Einflussfaktor ausgewiesen. Der positive Koeffizient zeigt, dass die Variable in der Ausprägung $\mathrm{y}_{\mathrm{h}}=1$ (Absolvent Fachhochschule) zu einer höheren Eintrittswahrscheinlichkeit der abhängigen Variablen führt. Fachhochschulabsolventen gründen somit eher in der Region als Universitätsab- 
Tab. 3 Schätzergebnisse

\begin{tabular}{|c|c|c|c|}
\hline & \multicolumn{3}{|c|}{ Logit abhängige Variable: Dummy Entfernung Hochschule/Gründung $=1$ bei $<50 \mathrm{~km}$} \\
\hline & (1) & $\begin{array}{l}\text { (2) Spin-offs: Gründung zwei Jahre } \\
\text { vor bis fünf Jahre nach Studium }\end{array}$ & $\begin{array}{l}\text { (3) Akademische Gründer: geringer zeitli- } \\
\text { cher Bezug zum Studium }\end{array}$ \\
\hline Jahre Abschluss/Gründung & $\begin{array}{l}-0,0439 * * \\
(0,069)\end{array}$ & $=$ & $=$ \\
\hline Hochschultyp $(\mathrm{FH}=1)$ & $\frac{1,4764 * * *}{(003)}$ & $\frac{1,3255^{* *}}{(0,016)}$ & $\frac{1,8708 * * *}{(0,001)}$ \\
\hline Forschungsintensität & $\underline{0,0006}$ & $\frac{-0,0004}{(0,801)}$ & $\frac{0,0030 * *}{(0,046)}$ \\
\hline Hochschulgröße & $\frac{0,0396^{* *} *}{\underline{(0,030)}}$ & $\frac{0,0402 *}{(0,058)}$ & $\frac{0,0380 * *}{(0,012)}$ \\
\hline $\begin{array}{l}\text { Dummy EXIST } \\
\text { Förderung }\end{array}$ & $\underline{0,1653}$ & $\underline{0,2099}$ & $\frac{-0,1152}{(0,745)}$ \\
\hline Faktor 1 Arbeitsmarkt & $\underline{0,1759}$ & $\underline{0,2199}$ & $\underline{0,0434}$ \\
\hline Faktor 2 Gründungsklima & $\frac{1,1996 * * *}{(0,001)}$ & $\frac{1,1078 * * *}{(0,009)}$ & $\frac{1,4014 * * *}{(0,000)}$ \\
\hline Infrastruktur & $\frac{-0,0312}{(0,302)}$ & $\frac{-0,0277}{(0,371)}$ & $\frac{-0,0531}{(0,250)}$ \\
\hline Konstante & $\frac{-1,4308^{*}}{(0,054)}$ & $\frac{-1,2891^{*}}{(0,090)}$ & $\frac{-1,4199 * *}{(0,048)}$ \\
\hline $\mathrm{N}^{\mathrm{a}}$ & 850 & 557 & 293 \\
\hline Pseudo $\mathrm{R}^{2}$ nach McFadden & $\underline{0,2071}$ & 0,1857 & 0,2505 \\
\hline
\end{tabular}

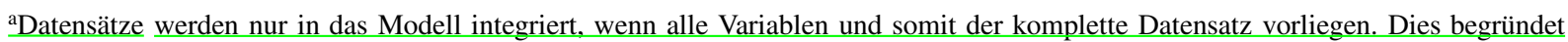
unterschiedliche Stichprobengrößen

Die Werte zeigen Beta-Regressionskoeffizienten ***Statistische Signifikanz bei $99 \%$ Konfidenzlevel, **Statistische Signifikanz bei $95 \%$ Konfidenzlevel, *Statistische Signifikanz bei $90 \%$ Konfidenzlevel, in den Klammern wird der $P$-Wert angeführt

solventen. Dies ist nachvollziehbar: Fachhochschulen sind in Forschur dd Lehre stärker auf die Region ausgerichtet, arbeiten verstärkt mit regionalen Unternehmen zusammen, verfügen in ländlichen Regionen über ein stärker regionales Einzugsgebiet (Kratz/Lenz 2N15) und bieten weniger spezialisierte Lehrangebote an $\backslash$ se/Hamm/Jäger et al. 2Q14: Jäger/Kopper 2214).

Die Hochs größe erweist sich in allen Modellen als signifikant. Je größer die Alma Mater ist, desto höher ist die Wahrscheinlichkeit, dass die Gründung innerhalb der Region erfolgt. Ein Erklärungsansatz ist die Tatsache, dass große Hochschulen über ein enges, in der Region verwurzeltes Kontaktnetz sowie über Labore und räumliche Infrastruktur verfügen. Zudem bieten große Hochschulen oft Gründungsberatungen und -zentren an, in denen sich Gründer ansiedeln können. ${ }^{11}$

Ein hochsignifikant usammenhang ist für die im Faktor Gründungsklima summierten Variablen „Anteil Selbstständige“, „Anteil Kleinstbetriebe“ und „Beschäftigungsquote“ zu erkennen. Liegt ein positives Gründungsklima vor - mit vielen Selbstständigen, Kleinstunternehmen und

11 Gegen den Einwand, dass große Hochschulen in Agglomerationsräumen häufiger auftreten und die Agglomeration der eigentliche Grund der hohen Verbleibsrate ist, spricht, dass die Einwohnerdichte als Teil des Faktors Arbeitsmarkt keinen signifikanten Einfluss zeigt.
Beschäftigten - ist nach Maßgabe der positiven Koeffizienten die Wahrscheinlichkeit einer regionalen Gründung signifikant höher. Dies bestätigen vorangegangene Studien (Lockett/Siegel/Wright et al. 2005). Eine hohe Zahl junger Kleinunternehmen gleicher o ähnlicher Sektoren fördert den Anschluss weiterer neuer Firmen (Iacobucci/Micozzi 2015: 440), erfolgreiche Gründer fungieren als Vorbild oder Ratgeber (Goldstein/Maier/Lugner 1895: 123).

Ebenso erwähnenswert sind die ht signifikanten Variablen, deren Resultate den Erwartungen widersprechen. So zeigt sich bei den Hochschulvariablen, dass Forschungsintensität und Gründungsorientierung keinen durchgängig signifikanten Einfluss auf die Standortwahl haben. Für beide Variablen wurde vermutet, dass Hochschulen als Inkubatoren besonders dann akademische Gründer in der Region halten, wenn sie forschungsaktiv und gründungsaffin sind. Eine Erklärung für das gegenteilige Ergebnis ist, dass diese Aspekte zwar die Gründungsaktivität an Hochschulen verstärken, jedoch nicht die Wahrscheinlichkeit des regionalen Verbleibs beeinflussen. Von Einfluss wird ebenso der Bezug zur Internetbranche sein, die von kostenschwerer Produktions- oder räumlicher Infrastruktur unabhängig ist. Es ist vorstellbar, dass Internetgründer nicht so sehr auf die Hochschule als Inkubator angewiesen sind wie Gründer anderer Branchen. Die Forschungsintensität zeigt lediglich in Modell 3 einen signifikanten Einfluss. Absolventen forschungs- 
starker Hochschulen. die lange vor bzw. nach Studienende gegründet haben, verbleiben demnach öfter in der Region.

Noch überraschender ist das Ergebnis der nicht signifikanten regionsseitigen Faktoren. Diese umfassen die Infrastruktur und die im Faktor Arbeitsmarkt subsumierten Variablen - Bruttoinlandsprodukt, Einwohnerdichte und die Quoten hochqualifizierter Arbeitnehmer bzw. im Dienstleistungsbereich Beschäftigter. Es lässt sich somit auf der Basis der Schätzungen kein systematischer Zusammenhang dieser Variablen mit der Verbleibsrate akademischer Gründer in der Region feststellen. Für den Faktor „Arbeitsmarkt“ wurde auf der Literaturbasis (Bruns/Görisch 2002: 37: Iacobucci/Micozzi 2015: 440) ein signifikanter Einfluss auf die Verbleibsquote erwartet und dass sich ein moderner, agglomerierter, dienstleistungsgeprägter und leistungsstarker (Arbeits-)Markt positiv auf den regionalen Verbleib der Gründer auswirkt. Die Resultate zeigen, dass lediglich im erweiterten Logit-Modell im Anhang, in dem Gründungen innerhalb Berlins ausgeschlossen wurden, ein schwach signifikanter Zusammenhang vorliegt. Dieses Ergebnis ist überraschend, verdeutlicht jedoch Potenziale: auch wenig agglomerierte Regionen mit wenig erfolgreichen Arbeitsmärkten können akademische Gründer zu einem vergleichbaren Anteil in der Region halten.

Zudem wurde erwartet. dass eine hohe Zentralität der Region sowie eine gute Verkehrsinfrastruktur die Standortwahl positiv beeinflusst. Obwohl die Variable „Infrastruktur" in anderen Studien und durchgeführten Einzelschätzungen der Logit-Modelle einen signifikanten Einfluss aufweist, erweist sie sich in den Gesamtmodellen als nicht signifikant. Es wird vermutet, dass ein Zusammenhang mit der Hochschulgröße besteht, der in den Einzelschätzungen zu verzerrten Resultaten führt, jedoch im Gesamtmodell aufgefangen wird. Eine potenzielle Erklärung für dieses Ergebnis liefern die Charakteristika der Internetbranche. Es werden keine Rohstoffe und Vorleistungen benötigt, die die Nähe zu einem infrastrukturell und anbindungstechnisch geeigneten Standpunkt in Kunden- und Zulieferernähe notwendig machen.

\section{Fazit}

Der Beitrag betrachtet die Standortwahl akademischer Gründer der Internetbrache und analysiert. welche hochschul- und regionsseitigen Faktoren ihre Wahl beeinflussen, innerhalb der Hochschulregion oder an einem anderen Standort zu gründen. Vor diesem Hintergrund wurde ein Datensatz auf der Basis von Gründerszene erstellt, eines digitalen Entrepreneur-Forums der Internetbranche. Die deskriptiven Ergebnisse zeigen zunächst eine starke Attrahierungskraft agglomerierter Zentren: Gründungen finden vor allem in Berlin. Hamburg. München und Köln statt.
Zydem zeigt sich, dass $40 \%$ der betrachteten Gründun2 innerhalb eines 50-km-Radius zu ihrer Alma Mater gründen, akademische Gründungen also eine erstaunliche Regionalwirksamkeit aufweisen - zumal Gründer der mobilen Internetbranche untersucht wurden. Während der höchste Anteil der Gründungen direkt im Jahr und den zwei Folgejahren des Studienendes erfolgt, erfolgt eine nicht zu vernachlässigende Zahl bereits vor dem Abschluss. Diese Start-ups werden zu einem überdurchschnittlichen Anteil in der Region gegründet. Dies könnte ein Argument sein, Spin-off-Förderung mit dem Ziel der Regionalwirksamkeit vermehrt auf potenzielle Gründer während des Studiums auszurichten.

Mithilfe der Regression wurde bezüglich der hochschulseitigen Determinanten gezeigt, dass eine Hochschule, die im Drittmittelerwerb erfolgreich und gründungsaffin ist, nicht automatisch dazu beiträgt, dass die umliegende Region verstärkt von ihren Spin-offs profitiert, denn für diese Variablen wurde kein signifikanter Zusammenhang mit dem Gründerverbleib festgestellt. Hochschultyp und -größe haben jedoch signifikanten Einfluss auf den regionalen Verbleib der Gründer. Absolventen von Fachhochschulen und großen Hochschulen verbleiben mit ihrer Gründung häufiger in der Region als Absolventen von Universitäten und kleiner Hochschulen. Ist die Förderung der Hochschulregion im Sinne der Third Mission ein strategisches Ziel, so ist dieses Ergebnis ein Argument für Fachhochschulen, mit ihrer Förderung vermehrt auf Gründungen durch Studierende und Absolventen zu setzen. Kleine Hochschulen haben es hingegen - vermutlich aufgrund fehlender Infrastruktur und eventuell auch Netzwerke in die regionale Wirtschaft tendenziell schwer, Absolventen und deren Gründungen in der Region zu halten.

Bezüglich der regionsseitigen Variablen wird deutlich, dass kein signifikanter Zusammenhang zwischen der Tertiärisierung, dem wirtschaftlichen Wohlergehen der Region gemessen an Bruttoinlandsprodukt, Einwohnerdichte, Quote Hochqualifizierter, Dienstleistungsquote und Verbreitung der Kreativen Klasse - mit dem Standortverbleib der betrachteten Gründer festgestellt wurde. Dieses unerwartete Ergebnis ist sicherlich zum Teil auf die Charakteristika der untersuchten Branche zurückzuführen, in der Gründer vergleichsweise mobil und räumlich ungebunden agieren. Auch Hochschulen in schwächeren Arbeitsmarktregionen können auf die Förderung von Spin-offs setzen, ohne mit außerordentlicher Abwanderung der Gründer in prosperierende Wirtschaftsregionen rechnen zu müssen. Während verdichtete, urbane Metropolen auch unabhängig von ihrer Eigenschaft als Hochschulstadt über ihre Grenzen hinaus hohe Attrahierungskraft auf Hochqualifizierte, Gründer und Kreative ausstrahlen, ist eine Hochschule mit hoher Regionalwirksamkeit und -orientierung speziell für kleine, 
peripher gelegene und wirtschaftsschwache Regionen eine wichtige Chance.

Die Ergebnisse zeigen, dass im Sinne einer nachhaltigen Regionalförderung bei der Frage, welcher Anteil akademischer Gründer innerhalb der Region verbleibt, das regionale Absorptionspotenzial eine Rolle spielt. Die Regression zeigt einen signifikanten Zusammenhang des Gründungsklimas und der Verbleibsquote der Gründer. In einer Region mit vielen Selbstständigen, Kleinstfirmen und geringer Arbeitslosigkeit entsteht offenbar eine gründungsaffine Atmosphäre, die eine hohe Anziehungskraft auf akademische Gründer ausübt. Die Abwägung, ob eine Hochschule mit dem Ziel der Regionalwirksamkeit auf die Förderung von Spin-offs setzt, sollte somit bei der Analyse der Gründungsatmosphäre beginnen, um die Regionalwirkung der Maßnahmen abschätzen zu können. Wird das Ziel verfolgt, die Regionalwirkung zu verbessern, sollten Hochschulakteure zusammen mit weiteren Handlungsträgern der Region auf förderliche Rahmenbedingungen und das Gründungsklima einwirken.

Zusammenfassend zeigt sich, dass akademische Gründer der Internetbranche - trotz ihrer sehr mobilen Grundvoraussetzungen - zu einem erstaunlich hohen Anteil in der Hochschulregion verbleiben. Es scheint dabei nicht zentral die Nähe zu Kunden, Zulieferern und die Unterstützung der Hochschule als Inkubator für den Verbleib von Gründern wichtig zu sein, interessanterweise auch nicht die Agglomerationsstufe und wirtschaftliche Prosperität der Region. Ein deutlicher Zusammenhang mit der regionalen Standortwahl ist jedoch für das regionale Gründungsklima zu erkennen. Dies weist darauf hin, dass eine Vernetzung von Gründern innerhalb der Region, das Vorhandensein einer „digital community“, also eines aktiven und vitalen wirtschaftlichen Gründungsklimas ein wichtiger Faktor in der Standortwahl von Gründern sein kann.

\section{Anhang}

Ergänzende Schätzergebnisse

Tab. 4 Ergänzende Schätzergebnisse

\begin{tabular}{|c|c|c|}
\hline & $\begin{array}{l}\text { Logit } \\
\text { Ausschluss Gründun- } \\
\text { gen in Berlin } \\
\text { abhängige Varia- } \\
\text { ble: Dummy Entfer- } \\
\text { nung Hochschule/ } \\
\text { Gründung = 1 bei } \\
\leq 50 \mathrm{~km}\end{array}$ & $\begin{array}{l}\text { OLS } \\
\text { Abhängige Variable: Rei- } \\
\text { sedistanz in km } \\
\text { Hochschule/Gründungsort }\end{array}$ \\
\hline $\begin{array}{l}\text { Jahre Ab- } \\
\text { schluss/ } \\
\text { Gründung }\end{array}$ & $\begin{array}{l}\frac{-0,4955^{*}}{(0,056)} \\
(0,05)\end{array}$ & $\begin{array}{l}2,9282 * \\
(0,094)\end{array}$ \\
\hline $\begin{array}{l}\text { Hochschultyp } \\
(\mathrm{FH}=1)\end{array}$ & $\frac{1,6759 * * *}{(0,001)}$ & $\frac{-174,5606^{* * * *}}{(0,000)}$ \\
\hline $\begin{array}{l}\text { FuE-Inten- } \\
\underline{\text { sität }}\end{array}$ & $\underline{0,0017}$ & $\frac{-0,0539}{(0,548)}$ \\
\hline $\begin{array}{l}\text { Hochschul- } \\
\text { größe }\end{array}$ & $\frac{(0,0375) * * *}{(0,005)}$ & $\frac{-5,2239 * * *}{(0,000)}$ \\
\hline $\begin{array}{l}\text { Dummy } \\
\text { EXIST }\end{array}$ & $\begin{array}{l}\underline{0,3208} \\
(0,350)\end{array}$ & $\frac{22,2385}{(0,305)}$ \\
\hline $\begin{array}{l}\text { Faktor } 1 \\
\text { Arbeits- } \\
\underline{\text { markt }}\end{array}$ & $\frac{0,2714^{*}}{\underline{(0,059)}}$ & $\frac{\sqrt{ } \sqrt{5}}{(0.502)}$ \\
\hline $\begin{array}{l}\text { Faktor } 2 \\
\text { Gründungs- } \\
\text { klima }\end{array}$ & $\frac{0.6888 * *}{(0,026)}$ & $\frac{-41.3107 * * *}{(0,002)}$ \\
\hline Infrastruktur & $\begin{array}{l}\frac{-0,0572}{(0,128)} \\
(0,128\end{array}$ & $\frac{-1,8002}{(0,520)}$ \\
\hline Konstante & $\begin{array}{l}\frac{-1,3537 * *}{(0,038)} \\
N=498 ; \\
\underline{\text { McFadden Pseudo }} \\
\underline{\mathrm{R}^{2}=0,1561}\end{array}$ & $\begin{array}{l}\underline{357.549 * * *} \\
\underline{(0,000)} \\
N=\underline{850 ;} \\
\text { korrigiertes } \mathrm{R}^{2}=\underline{0.0932}\end{array}$ \\
\hline
\end{tabular}

***Statistische Signifikanz bei $99 \%$ Konfidenzlevel, **Statistische Signifikanz bei $95 \%$ Konfidenzlevel. *Statistische Signifikanz bei $90 \%$ Konfidenzlevel; Logit: 2D-Cluster-Ansatz mit adjustierten. OLS-Schätzung mit robusten Standardfehlern, in den Klammern wird der $P$-Wert angeführt

\section{Logit-Schätzansatz:}

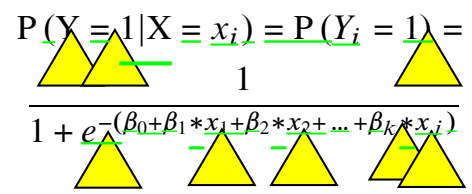

$\mathrm{P}=$ Wahrscheinlichkeit der Gründung innerhalb der Hochschulregion (50-km-Radius um Hauptstandort der Hochschule)

$\mathrm{x}=$ erklärende Variable

$\mathrm{Y}=$ abhängige Variable

$\mathrm{j}=1, \ldots, \mathrm{i}(\mathrm{i}=$ Anzahl Beobachtungen $)$ 


$$
\begin{aligned}
& \mathrm{k}=1, \ldots, n(n=\text { Anzahl Variablen }) \\
& \beta=\text { Regressionskoeffizient } \\
& \underline{u_{\mathrm{k}}}=\text { Residualgrößen }
\end{aligned}
$$

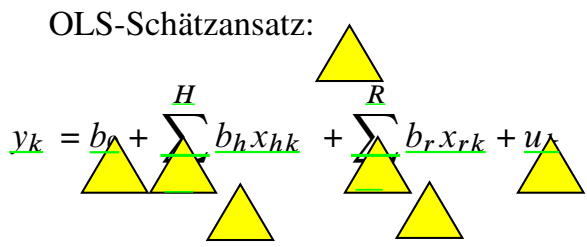

$\mathrm{y}_{\mathrm{k}}=$ Reisedistanz Alma Mater-Gründungsstandort in $\mathrm{km}$

$\mathrm{b}=$ konstantes Glied

$\mathrm{x}_{\mathrm{hk}}=$ hochschulseitige Variablen

$\mathrm{x}_{\mathrm{rk}}=$ regionsseitige Variablen

$\mathrm{k}=$ Beobachtung

\section{Literatur}

Audretsch, D.B.; Falck, O.; Feldman, M.P.; Heblich, S. (2012): Local Entrepreneurship in Context. In: Regional Studies 46, 3, 379-389.

Backes-Gellner, U.; Demirer, G.; Sternberg, R. (2002): Individuelle und regionale Einflussfaktoren auf die Gründungsneigung von Hochschülern. In: Schmude, J.; Leiner, R. (Hrsg.): Unternehmensgründungen - Interdisziplinäre Beiträge zum Entrepreneurship Research. Heidelberg, 63-97.

Backhaus, K.; Erichson, B.; Plinke, W.; Weiber, R. (2011): Multivariate Analysemethoden. Eine anwendungsorientierte Einführung. Berlin, Heidelberg.

Beyer, F.; Majer, B. (2002): Wege der Abgrenzung von Wirkungsregionen der Universität. In: Bathelt, H.; Schamp, E. (Hrsg.): Die Universität in der Region. Frankfurt, 9-16. = Frankfurter Wirtschafts- und Sozialgeographische Schriften 71.

Blum, U.; Leibbrand, F. (2001): Entrepreneurship und Unternehmertum - Denkstrukturen für eine neue Zeit. Wiesbaden.

BMWI - Bundesministerium für Wirtschaft und Technologie (2008): Rahmenbedingungen und Ausprägung der akademischen Gründungsförderung an 100 deutschen Fachhochschulen. Berlin. = Forschungsbericht 576.

BMWI - Bundesministerium für Wirtschaft und Technologie (2012): Monitoring Report Digitale Wirtschaft 2012 - Mehrwert für Deutschland. Berlin.

Bruns, R. W.; Görisch, J. (2002): Unternehmensgründungen aus Hochschulen im regionalen Kontext - Gründungsneigung und Mobilitätsbereitschaft von Studierenden. Karlsruhe. = Arbeitspapiere Unternehmen und Region R1/2000.

Bundesagentur für Arbeit (2015): Sonderauswertung Sozialversicherungspflichtig Beschäftigte nach Wirtschaftszweigen WZ 2008. Nürnberg.

Caniëls, M.; van den Bosch, H. (2011): The Role of Higher Education Institutions in building regional innovation systems. In: Papers in Regional Science 90, 2, 271-286.

Di Gregorio, D.; Shane, S. (2003): Why do some universities generate more start-ups than others? In: Research Policy 32, 2, 209-227.

Dinse, H.-C.; Hamm, R.; Jäger, A.; Karl, H.; Kopper, J.; Strotebeck, F.; Warnecke, C. (2014): Regionale Transfereffekte verschiedener Hochschultypen (Abschlussbericht). Bochum. = Beiträge zur Ballungsraumforschung 14.

Egeln, J.; Gottschalk, S.; Rammer, C. (2004): Location decisions of spin-offs from public research institutions. In: Industry and Innovation $11,3,207-223$.
Egeln, J.; Gottschalk, S.; Rammer, C.; Spielkamp, A.(2002): SpinoffGründungen aus der öffentlichen Forschung in Deutschland. Bonn. = Bundesministerium für Bildung und Forschung EXISTStudien 4.

Florida, R. (20 The rise of the Creative Class and how it's transforming work, leisure, community and everyday life. New York.

Fritsch, M. (2016): Entrepreneurship. Theorie, Empirie, Politik. Wiesbaden.

Fritsch, M.; Aamoucke, R. (2013): Regional public research, higher education, and innovative start-ups: an empirical investigation. In: Small Business Economics 41, 4, 865-885.

Fritsch, M.; Henning, T.; Slavtchev, V.; Steigenberger, N. (2007): Hochschulen, Innovation, Region. Wissenstransfer im räumlichen Kontext. Berlin.

Fritsch, M.; Schroeter, A. (2011): Why does the effect of new business formation differ across regions? In: Small Business Economics $36,4,383-400$.

Fromhold-Eisebith, M. (1992): Wissenschaft und Forschung als regionalwirtschaftliches Potential? Das Beispiel von Rheinisch Westfälischer Technischer Hochschule und Region Aachen. Aachen.

Goldstein, H. A. (2005): The Role of Knowledge Infrastructure in Regional Economic Development: The Case of the Research Triangle. In: Canadian Journal of Regional Science 28, 2, 199-220.

Goldstein, H. A. (2009): What we know and what we don't know about the regional economic impacts of universities. In: Varga, A. (Hrsg.): Universities, knowledge transfer and regional development. Cheltenham, 11-35.

Goldstein, H.; Maier, G.; Lugner, M. (1995): The university as an instrument for economic and business development: U.S. and European comparisons. In: Dill, D. D.; Sporn, B. (Hrsg.): Emerging patterns of social demand and university reform: through a glass darkly. Oxford, 105-133.

Gottschalk, C.; Hamm, R.; Imöhl, I. (2010): Die Bedeutung der Kreativen Klasse für die wirtschaftliche Entwicklung der nordrhein-westfälischen Regionen. https://www.hs-niederrhein. de/fileadmin/dateien/institute/niers/pdf/Kreative-Klasse. Abschlussbericht-Teil 1.pdf (29.06.2016).

Hamm, R.; Jäger, A.; Karl, H.; Kopper, J.; Strotebeck, F.; Warnecke, C. (2013a): Regionale Transfereffekte verschiedener Hochschultypen. Analysen und Strategien für eine verbesserte Potenzialausschöpfung - Zwischenbericht II. https://www.hs-niederrhein.de/ fileadmin/dateien/institute/niers/pdf/Publikationen/REGTRANS. 2.ZWIBI.28.10.pdf (15.07.2016).

Hamm, R.; Jäger, A.; Karl, H.; Kopper, J.; Strotebeck, F.; Warnecke, C. (2013b): Regionale Transfereffekte verschiedener Hochschultypen. Analysen und Strategien für eine verbesserte Potenzialausschöpfung - Zwischenbericht I. https://www.hs-niederrhein.de/ fileadmin/dateien/institute/niers/pdf/Publikationen/REGTRANS_ ZWIBI 01.pdf (16.07.2016)

Hemer, J.; Walter, G.; Berteit, H.; Göthner, M. (2006): Erfolgsfaktoren für Unternehmensausgründungen aus der Wissenschaft. Karlsruhe.

Heumann, S. (2010): Bewegliche Ziele - Die räumlich-strategische Differenzierung der akademischen Gründungsförderung an 50 deutschen Universitäten. In: Beiträge zur Hochschulforschung 32, 3, 54-77.

Iacobucci, D.; Micozzi, A. (2015): How to evaluate the impact of academic spin-offs on local development: an empirical analysis of the Italian case. In: The Journal of Technology Transfer 40, 3, 434-452.

INCHER - International Centre for Higher Education Research Kassel (2011): KOAB Absolventenbefragung 2011, Jahrgang 2009. Kassel.

Jäger, A.; Kopper, J. (2014): Third mission potential in higher education: measuring the regional focus of different types of HEIs. In: Review of Regional Research 34, 2, 95-118. 
Koschatzky, K. (2002): Hochschulen impregionalen Gründungskontext. In: Koschatzky, K.; Kulicke, M. sg.): Wissenschaft und Wirtschaft im regionalen Gründungskontext. Stuttgart, 31-36.

Kratz, F.; Lenz, T. (2015): Regional-ökonomische Effekte von Hochschulabsolventen. In: Beiträge zur Hochschulforschung 37, 2, 8-28.

Krebs, D. (1995): Selbstselektion: demographisches oder attitudinales Problem? In: ZA-Information/Zentralarchiv für empirische Sozialforschung 36, 114-125.

Kulicke, M.; Dornbusch, F.; Kripp, K.; Schleinkofer, M. (2012): Nachhaltigkeit der EXIST-Förderung. Gründungsunterstützung an Hochschulen, die zwischen 1998 und 2011 gefördert wurden. Bericht der wissenschaftlichen Begleitforschung zu „EXIST Existenzgründungen aus der Wissenschaft“. Karlsruhe.

Landry, R.; Amara, N.; Rherrad, I. (2006): Why are some university researchers more likely to create spin-offs than others? Evidence from Canadian universities. In: Research Policy 35, 10, $1599-1615$.

Lockett, A.; Siegel, D.; Wright, M.; Ensley, M. D. (2005): The creation of spin-off firms at public research institutions: Managerial and policy implications. In: Research Policy 34, 7, 981-993.

Möller, K. (2014): Culturally clustered or in the cloud? Location of internet start-ups in Berlin. London. $=$ Spatial Economic Research Centre Discussion Paper 157.

Neubecker, J. (2006): Finanzierung durch Corporate Venture Capital und Venture Capital. Empirische Untersuchung zum Value Added junger, innovativer Unternehmen in Deutschland. Wiesbaden.

Petersen, M. A. (2009): Estimating Standard Errors in Finance Panel Data Sets: Comparing Approaches. In: The Revue of Financial Studies 22, 1, 435-480.

Roessler, I.; Duong, S.; Hachmeister, C.-D. (2015): Welche Missionen haben Hochschulen? Third Mission als Leistung der Fachhochschulen für die und mit der Gesellschaft. Gütersloh. = Centrum für Hochschulentwicklung Arbeitspapier 182.
Schefczyk, M. (2004): Erfolgsstrategien deutscher Venture Capital-Gesellschaften. Analyse der Investitionsaktivitäten und des Beteiligungsmanagements von Venture Capital-Gesellschaften. Stuttgart. $=$ Betriebswirtschaftliche Abhandlungen 106.

Schleinkofer, M. (2006): Unternehmensgründungen aus Hochschulen und außeruniversitären Forschungseinrichtungen. Empirisch basierte Studie zu Erfolgsfaktoren aus dem betrieblichen Umfeld. Diplomarbeit an der Universität Regensburg.

Schmidt, A.; Heinrichs, S.; Walter, A. (2010): Ein Forschungsüberblick zu Einflussgrößen der Entwicklung technologieorientierter Spinoff-Unternehmen. Kiel. = Arbeitspapiere Institut für Betriebswirtschaftslehre, Universität Kiel.

Schmitt, N. (1996): Uses and abuses of coefficient alpha. In: Psychological Assessment 8, 4, 350-353.

Schmoch, U.; Licht, G.; Reinhard, M. (2000): Wissens- und Technologietransfer in Deutschland. Studie im Auftrag des Bundesministeriums für Bildung und Forschung. Karlsruhe, Mannheim, München.

Schmude, J. (1994): Geförderte Unternehmensgründungen in BadenWürttemberg. Eine Analyse der regionalen Unterschiede des Existenzgründungsgeschehens am Beispiel des Eigenkapitalhilfe-Programms (1979-1989). Stuttgart. = Erdkundliches Wissen 114.

Statistisches Bundesamt (2013): Monetäre hochschulstatistische Kennzahlen - Einzelne Hochschulen. Spezialauswertung Fachserie 11/4.3.2. Wiesbaden.

Wissenschaftsrat (2007): Empfehlungen zur Interaktion von Wissenschaft und Wirtschaft. Oldenburg.

Zhang, J. (2006): Silicon Valley's regional advantages: evidence from venture capital data. In: Karlsson, C.; Johannson, B.; Stough, R. R. (Hrsg.): Entrepreneurship and Dynamics in the Knowledge Economy. New York, 167-188. 